\title{
Up-regulation of S100A16 expression promotes epithelial-mesenchymal transition via Notch1 pathway in breast cancer
}

Wenbin Zhou ${ }^{1+}$, Hong Pan ${ }^{1 \dagger}$, Tiansong Xia ${ }^{1}$, Jinqiu Xue ${ }^{1}$, Lin Cheng ${ }^{1}$, Ping Fan ${ }^{2}$, Yifen Zhang ${ }^{3}$, Weidong Zhu ${ }^{4}$, Yi Xue ${ }^{5}$, Xiaoan Liu', Qiang Ding ${ }^{1}$, Yun Liu ${ }^{5^{*}}$ and Shui Wang ${ }^{1^{*}}$

\begin{abstract}
Background: Our previous studies demonstrated that S100A16 promotes adipogenesis and is involved in weight gain attenuation induced by dietary calcium. Till now, the function of S100A16 in the breast cancer remains to be elucidated.

Results: In this study, we observed that S100A16 was expressed in higher levels in human breast cancer tissues compared with paired adjacent non-cancerous tissues. Further examination showed that overexpression of S100A16 in MCF-7 cells could increase cell proliferation and colony formation. One major mechanistic change was that S100A16 was able to up-regulate the transcription factors Notch1, ZEB1, and ZEB2, which had the capacities to directly repress the expression of epithelial markers $\mathrm{E}$-cadherin and $\mathrm{\beta}$-catenin but increase mesenchymal markers $\mathrm{N}$-cadherin and vimentin, a characterized phenotype of epithelial-mensenchymal transition (EMT). In addition to display with morphologic change, migration and invasion were increased in S100A16 over-expressed MCF-7 cells. Importantly, knockdown of Notch1 by specific siRNA could reverse the EMT induced by S100A16 overexpression, which confirmed that Notch1 played a critical role in the process of EMT induced by S100A16.
\end{abstract}

Conclusions: All together, our data indicated that S100A16 had a potential function to regulate some embryonic transcription factors to promote EMT in breast cancer cells which may be an important target site for the therapy of breast cancer.

Keywords: Breast cancer, S100A16, EMT, Notch1, MCF-7

\section{Background}

Breast cancer is a worldwide malignant disease. Although disease-free survival and overall survival have been significantly improved, there are still some parts of patients diagnosed with metastases after systemic therapies [1]. The poor survival of advanced breast cancer is related to the lack of effective therapies, which can be attributed to poor understanding of the molecular mechanisms underlying the progression of disease toward invasion and metastasis $[2,3]$. Up to now, many molecular markers have been found, such as estrogen receptor (ER), progesterone

\footnotetext{
* Correspondence: liuyun@njmu.edu.cn; ws0801@hotmail.com

${ }^{\dagger}$ Equal contributors

${ }^{5}$ Department of Geratology, The First Affiliated Hospital with Nanjing Medical University, 300 Guangzhou Road, 210029 Nanjing, China

'Department of Breast Surgery, The First Affiliated Hospital with Nanjing

Medical University, 300 Guangzhou Road, 210029 Nanjing, China

Full list of author information is available at the end of the article
}

receptor (PR) and HER2. ER and/or PR positive are considered as hormone receptor positive $(\mathrm{HR}+)$, which is a predictor of endocrine therapy and good clinical outcome. HER2 is an indicator of targeted therapy and a predictor of poor clinical outcome. However, there is huge desire to improve the survival of advanced breast cancer. Therefore, it is important to identify new predictive biomarkers, especially those indicators for invasiveness of the disease.

S100A16 is a novel low-affinity calcium-binding protein of the EF-hand superfamily [4-6]. Our previous studies suggest that S100A16 promotes adipogenesis and is involved in weight gain attenuation induced by dietary calcium $[7,8]$. Importantly, S100A16 is a ubiquitously expressed protein, which is up-regulated in various malignant tumors [5,9]. However, the physiological functions of S100A16 in malignant tumors are still undefined.

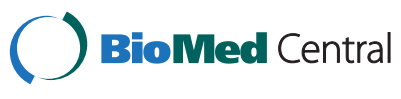

(c) 2014 Zhou et al.; licensee BioMed Central Ltd. This is an Open Access article distributed under the terms of the Creative Commons Attribution License (http://creativecommons.org/licenses/by/4.0), which permits unrestricted use, distribution, and reproduction in any medium, provided the original work is properly credited. The Creative Commons Public Domain Dedication waiver (http://creativecommons.org/publicdomain/zero/1.0/) applies to the data made available in this article, unless otherwise stated. 
The epithelial-mesenchymal transition (EMT) plays a crucial role in the formation and differentiation of multiple tissues. Importantly, it is clear that EMT is involved in metastatic events in cancer and highly relevant to tumor progression [10]. Notch signaling pathway affects cancer patient survival, especially for Notch1 and Notch4 receptors [11]. Activation of the Notch signaling pathway participates in EMT induction [12,13].

All of these findings imply that S100A16 may be involved in the progression of breast cancer. To investigate the function of S100A16 in breast cancer, we first documented here that S100A16 was over-expressed in breast cancer tumors. Further examination demonstrated that up-regulation of S100A16 promoted EMT via Notch1 pathway in breast cancer cell line MCF-7. Our results suggested that S100A16 may be involved in the progress of breast cancer.

\section{Methods}

RNA extraction, reverse transcription and quantitative RT-PCR

The Trizol reagent (Invitrogen) was used to isolate total RNA following manufacturer's instructions. Then RNA was used for cDNA synthesis using a reverse transcriptase reaction kit (TaKaRa). Quantitative RT-PCR for $\beta$-actin and other genes was performed for every cDNA sample. All PCR reactions were performed using the fluorescent SYBR Green I methodology. Real-time quantitative PCR was performed with SYBR Premix Ex Taq (TaKaRa) according to the manufacturer's instructions. $\beta$-actin was used as a loading control.

S100A16 expression was measured in 20 breast cancer tissue samples compared with paired adjacent noncancerous tissue using qRT-PCR. The paired noncancerous tissues were adjacent non- cancerous tissue, which were at least $5 \mathrm{~cm}$ away from the cancerous tissue, which were normal "histologically". This study was approved by the ethics commit of the First Affiliated Hospital with Nanjing Medical University. All patients provided written informed consent for their clinical information to be reviewed by us. And it was in compliance with the Helsinki Declaration.

\section{Cell culture}

The human breast cancer cell lines MCF-7, T47D, BT474, ZR-75-1, MDA-MB-231, BT549, MCF10A, 184A1 and 184B5 were obtained from the American Tissue Culture Collection (ATCC), and cultured in complete medium (DMEM, IMEM or 1640 supplemented with $10 \%$ fetal bovine serum) in a $5 \% \mathrm{CO}_{2} 37^{\circ} \mathrm{C}$ incubator.

\section{Plasmid construction and lentivirus packaging}

S100A16 gene was cloned by RT-PCR from RNA of human MCF-7 cells and cloned into the pLV-GFP vector (a gift from Dr. Beicheng Sun, Nanjing Medical University, China) using BamHIand MluI sites, named pLV-S100A16. Plasmid sequencing was performed by Invitrogen Company with a 3730 DNA analyzer. Primer sequences for S100A16: Forward: TTGGATCCGGAGATGTCAGACTGCTACAC, Reverse: TTACGCGTAAAGGGGTCTCTAGCTGCTG.

Recombinant lentivirus was generated from $293 \mathrm{~T}$ cells using calcium phosphate precipitation. The levtivirus was then transduced into MCF-7 and T47D cells mediated by polybrene $(8 \mu \mathrm{g} / \mathrm{ml})$. Plain pLV-GFP lentivirus infected MCF-7 and T47D cells were used as the control.

\section{Colony formation assay}

Cells used for colony formation analysis were seeded into 6-well plates $(1000$ cells $/ \mathrm{ml})$ and cultured normally for two weeks. The colonies were fixed in paraform and stained with Giemsa after washed with PBS twice. The number of colonies was counted.

\section{MTT assay}

MTT assay was applied to assess cell proliferation as described previously [14]. Cell proliferation was compared at five time point $(1,2,3,4$, and 5 days) after the cells were seeded. The absorbance of the samples was measured at $490 \mathrm{~nm}$ on a scanning multi-well spectrophotometer. Each experiment was repeated three times.

\section{Cell migration and invasion assay}

In vitro cell migration and invasion assays were performed as described previously [15]. Cells growing in the log phase were trypsinized, re-suspended in serum-free medium, and seeded into Boyden chamebers $(8 \mu \mathrm{m}$ pore size with polycarbonate membrane). The chambers were then inserted into transwell apparatus (Costar, Cambridge, MA, USA). The chambers were coated with Matrigel (BD Biosciences, San Jose, USA) when cell invasion assay was done. And the chambers were coated without Matrigel when cell migration assay was done. Medium with 10\% FBS $(600 \mu \mathrm{l})$ was added to the lower chamber. After incubation of $24 \mathrm{~h}$, cells on the top surface of the insert were removed by wiping with cotton swab. Cells that migrated to the bottom surface of the insert were stained in $0.1 \%$ crystal violet for $30 \mathrm{~min}$, rinsed in PBS and then subjected to microscopic inspection. Images of three random fields $(10 \times)$ were captured from each membrane, and the number of migratory or invasive cells was counted. Triplicate assays were used for each experiment.

\section{Notch1 small interfering RNA transfection}

Notch1 siRNA were purchased from GenePharma (Shanghai, China). MCF7-S10016 cells were transfected with Notch1 siRNA as described previously [14]. The sequences of Notch1 were GUC CAG GAA ACA ACU 
GCA ATT (sense) and UUG CAG UUG UUU CCU GGA CTT (antisense), respectively. The sequences of negative control were UUC UCC GAA CGU GUC ACG UTT (sense) and ACG UGA CAC GUU CGG AGA ATT (antisense), respectively.

\section{Western blot analysis}

Western blot analysis was performed as described previously [14]. Monoclonal anti-S100A16 (R\&D), ZEB1 (Santa Cruz), ZEB2, Notch1 (Abcam), $\beta$-Catenin, NCadherin, E-cadherin, Vimentin were purchased. GAPDH (Santa Cruz) was used to as loading control. The intensity of the bands was determined by using densitometric analysis. All experiments were performed three times.

\section{Immunohistochemistry and Fluorescence in situ hybridization}

Breast tissue samples were obtained at the time of surgery. Immunohistochemistry (IHC) analyses were performed on 4. $\mu \mathrm{m}$, formalin-fixed, paraffin-embedded slides from breast cancer tissues and matched non-cancerous tissues. Paraffin-embedded tissue sections were deparaffinized, rehydrated, rinsed, and immersed in $10 \mathrm{mM}$ sodium citrate $(\mathrm{pH}$ 6.0) for antigen retrieval under high pressure in a pressure cooker for 3 minutes. After treated with in methanol containing 3\% hydrogen peroxide for $10 \mathrm{~min}$ to block endogenous peroxidase activity, the slides were incubated with primary antibody for 1 hour at $37^{\circ} \mathrm{C}$. After washing, sequential incubations were performed with horseradish peroxidase (HRP) conjugated antibodies (Invitrogen) for $30 \mathrm{~min}$ at room temperature. The stain was visualized using DAB Plus (Dako) and hematoxylin counterstain. The expressions of S100A16, ER, PR, HER2 were all detected by IHC.

A Fluorescence in situ hybridization (FISH) test for HER2 gene amplification was routinely ordered when HER2 was IHC 2+. FISH was performed using the PathVysion HER2 DNA FISH Kit (Vysis Inc, Downers Grove, IL), according to the manufacturer's instructions.

\section{Immunofluorescence}

Cells were seeded in a 6-well culture plate. The cells were immunofluorescence-labeled according to the manufacturer's instructions. Cells were incubated with a blocking buffer for 1 hour to suppress non-specific binding after washing and fixing. And then, cells were incubated with primary antibody (ZEB1, ZEB2, $\beta$ Catenin, N-Cadherin, E-cadherin, Vimentin) at $4^{\circ} \mathrm{C}$ overnight, followed by incubation with a secondary antibody for 1 hour. Cells were further mounted with DAPI (4', 6-diamidino-2-phenylindole) for 5 minutes and analyzed using fluorescence microscopy.

\section{Luciferase reporter assay}

We analyzed the putative promoter region of Notch1 $(-1998$ to +76$)$ using a luciferase reporter assay. The Notch1 promoter was cloned by PCR from genomic DNA of human MDA-MB-231 cells. PCR-based cloning was used to generate different segments $(-483$ to $+76,-998$ to $+76,-1496$ to +76 , and -1998 to +76 ) of the Notch-1 gene promoter. Then the amplified products were ligated into the pGL3 basic vector using NheI and XhoI sites (New England Biolab, UK), respectively, named pGL3-Notch1-I, pGL3-Notch1-II, pGL3-Notch1-III, and pGL3-Notch1-IV. Plasmid sequencing was performed by Invitrogen Company (China) with a 3730 DNA analyzer. Primer sequences for Notch-1 promoter cloning:

Forward 1:5'-ACTTGCTAGCGAGAAGTAGTCCCA GGCGC-3',

Forward 2:5'-CCGGGCTAGCGGAGGACGGTGAC CGAG-3',

Forward 3:5'-GGCTGCTAGCAGAGTGCTCAGCA TTTGGC-3',

Forward 4:5' -ACCAGCTAGCACCCCCTATCCAGG GATC-3',

Reverse: 5'-TGGGCTCGAGCCTACCTCGTGCGGCG -3' .

For the S100A16 responsive promoter activity, S100A16-transduced MCF-7 cells, MCF7-S100A16 cells were chosen to analyze the putative promoter region of Notch1 (-1998 to +76) using dual luciferase reporter assay. MCF7-S100A16 cells were seeded into 6-well culture plates 1 day prior to transient transfection. Plasmids pGL3-Notch1-I, pGL3-Notch1- $\alpha$, pGL3-Notch1$\beta$, and pGL3-Notch1-Ic were respectively co-transfected with the Renilla luciferase expression plasmid into MCF7S100A16 cells. After $48 \mathrm{~h}$ transfection, cells were collected and luciferase activity was measured using the Dual Luciferase Reporter Assay System (Promega, USA) according to the manufacturer's instructions. Then, the cells were harvested and processed for dualluciferase reporter activity, in which the firefly luciferase activity was normalized by renilla luciferase activity.

\section{Statistical analysis}

All experiments in this study were repeated in triplicate. Paired $t$-test was applied to calculate the statistical significance of the expression levels of S100A16 mRNA in breast cancer tissue and adjacent noncancerous tissue. Student $t$ test was applied to calculate the statistical significance of other experimental results. A significant difference was concluded for $P<0.05$. 


\section{Results}

\section{S100A16 was overexpressed in human breast} cancer tissues

We measured S100A16 expression in 20 breast cancer tissue samples compared with paired adjacent noncancerous tissue using qRT-PCR. The clinical characteristics of all subjects are summarized in Table 1 . Of these 20 paired samples, 14 showed significantly higher S100A16 mRNA expression in the cancer tissue compared with the adjacent tissue (Figure 1A). There was no significant difference of S100A16 mRNA levels between subgroups of breast cancer with different ER, PR or HER2 status (data not shown). The mean expression level of S100A16 mRNA in breast cancer tissue was also significantly higher than that in adjacent non-cancerous tissue using a scatter plot (Figure 1B). Besides, Immunostainings for S100A16 were performed in breast cancer tissues and the matched non-cancerous tissues. Interestingly, over-expression of S100A16 was observed particularly in the invasive front in breast cancer tissues (Figure 1D), which indicated that S100A16 might be related to EMT. To further study the expression of S100A16 in breast cancer, S100A16 protein

Table 1 Characteristics of the $\mathbf{2 0}$ patients with breast cancer

\begin{tabular}{|c|c|c|}
\hline \multirow[t]{2}{*}{ Variables } & \multicolumn{2}{|l|}{ Cases } \\
\hline & No. & $\%$ \\
\hline Ages (mean, range) & $56,37 \sim 70$ & \\
\hline$\leq 50$ & 7 & 35 \\
\hline$>50$ & 13 & 65 \\
\hline \multicolumn{3}{|l|}{ Tumor size } \\
\hline$\leq 2 \mathrm{~cm}$ & 9 & 45 \\
\hline$>2 \mathrm{~cm}$ & 11 & 55 \\
\hline \multicolumn{3}{|c|}{ Lymph node involvement } \\
\hline Negative & 11 & 55 \\
\hline Positive & 9 & 45 \\
\hline \multicolumn{3}{|c|}{ Estrogen receptor status } \\
\hline Negative & 8 & 40 \\
\hline Positive & 12 & 60 \\
\hline \multicolumn{3}{|c|}{ Hormone receptor status } \\
\hline Negative & 7 & 35 \\
\hline Positive & 13 & 65 \\
\hline \multicolumn{3}{|l|}{ HER2 status } \\
\hline Negative & 14 & 70 \\
\hline Positive & 6 & 30 \\
\hline \multicolumn{3}{|l|}{ Molecular subtype } \\
\hline $\mathrm{HR}+/ \mathrm{HER} 2-$ & 10 & 50 \\
\hline Triple negative & 4 & 20 \\
\hline HER2+ & 6 & 30 \\
\hline
\end{tabular}

$\mathrm{HR}+$, ER and/or PR positive. expression was detected by Western blot in eight human breast cancer cell lines versus three normal breast epithelial cell lines (MCF10A, 184A1 and 184B5). It was expressed in two ER positive cell lines MCF-7 and ZR-75-1 (ER positive and HER2 negative cell lines) (Figure 1C) and two ER negative cell lines MDA-MB468 (triple negative cell line) and SK-BR3 (HER2 amplified cell line) (Figure 1C). Additionally, lower expression level of S100A16 was also detected in BT474 (ER positive and HER2 overexpression cell line) and MCF10A cells (Figure 1C). S100A16 protein expression was not detected in other two normal breast epithelial cell lines 184A1 and 184B5 (Additional file 1: Figure S1). Among these limited cell lines, there was no direct correlation between S100A16 and ER levels or HER2 expression although relative levels of S100A16 in MDA-MB-468 and SK-BR3 were higher compared with expressed ER positive cells (Figure 1C).

\section{Up-regulation of S100A16 increased the capacities of migration and invasion in MCF-7 and T47D cells}

The results of clinical samples indicated that S100A16 may be associated with aggressive behavior in breast cancer (Figure 1A and B). To validate this, we overexpressed S100A16 using pLV-S100A16 lentivirus in MCF-7 and T47D cells. The protein levels of S100A16 were elevated after infection with pLV-S100A16 lentivirus (Figure 2A and Additional file 1: Figure S2). The new cell lines were named as MCF7-S100A16 and T47D-S100A16, and the control cell lines were named as MCF7-GFP and T47D-GFP.

The cell proliferation rate was increased after S100A16 overexpression (Figure 2B). And up-regulation of S100A16 markedly increased the number of MCF-7 cell colonies (Figure 2C). Furthermore, up-regulation of S100A16 increased cell migration (Figure 2D and Additional file 1: Figure S3) and invasion (Figure 2E and Additional file 1: Figure S4) abilities compared with control cells, which were evaluated by specific transwell chambers. All of these results demonstrated that up-regulation of S10016A made cells in an aggressive phenotype.

\section{Overexpression of S100A16 promoted EMT in MCF-7 and T47D cells}

S100A16 over-expressed MCF-7 cells displayed morphologic changes with a spindle-like shape (Figure $3 \mathrm{~A}$ ) (similar changes were founded for T47D-S100A16 cells, data not shown). Up-regulation of S100A16 also disrupted E-cadherin mediated cell-cell adhesion. We observed that epithelial markers E-cadherin and $\beta$ Catenin were significantly reduced in mRNA and protein levels in MCF7-S100A16 cells compared with MCF7-GFP cells (Figure 3B and C) and T47D-GFP cells (Additional file 1: Figure S2). In contrast, 


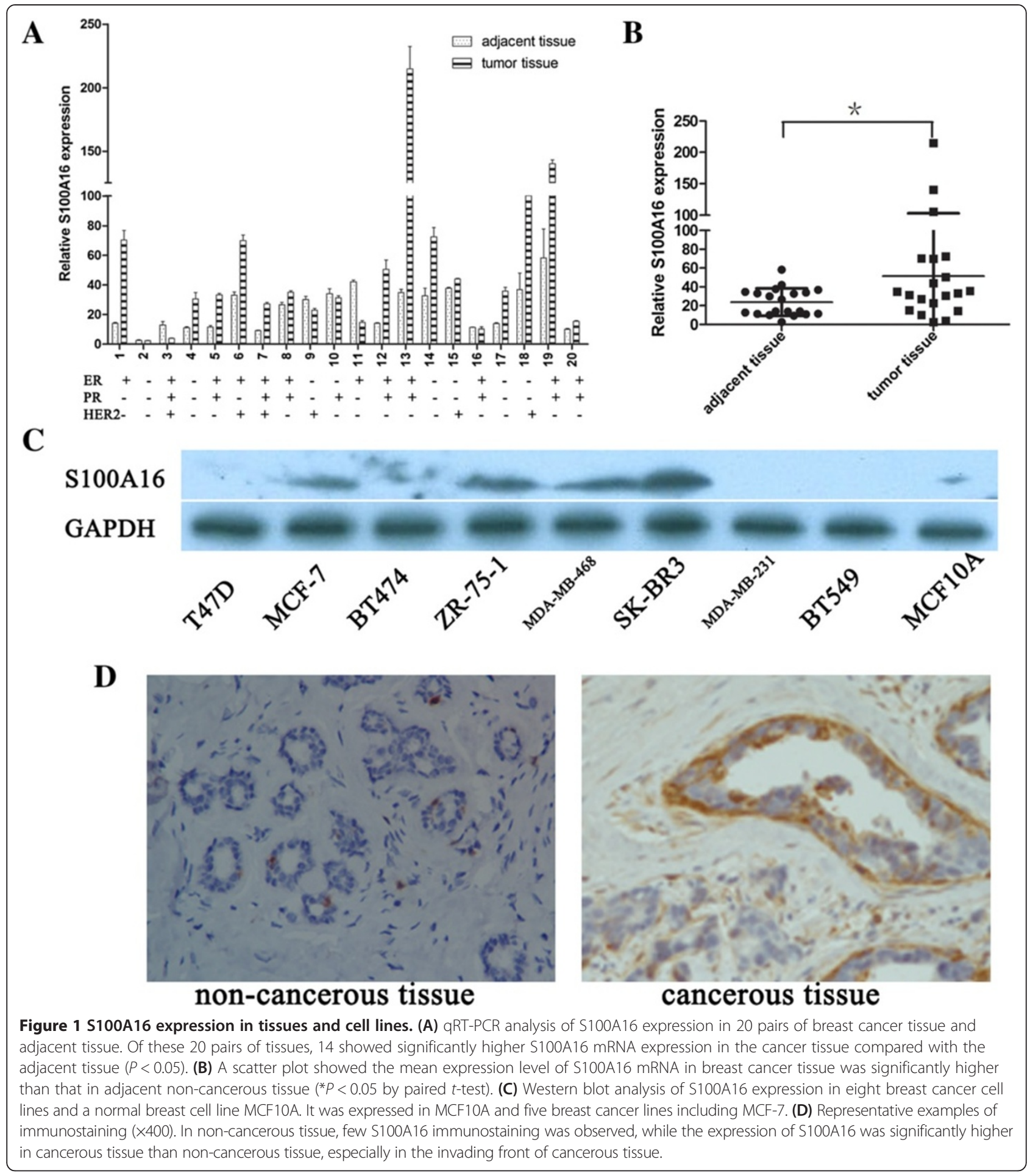

mesenchymal markers Vimentin and N-cadherin were significantly up-regulated in MCF7-S100A16 cells (Figure 3B and C) and T47D-S100A16 cells (Additional file 1: Figure S2), indicating a characterized feature of EMT.

Consistently, the epithelial and mesenchymal markers were also examined by immunofluorescence staining. Both E-cadherin and $\beta$-catenin resided in the cell membrane and the intensity of fluorescence were reduced in MCF7-S100A16 cells compared with MCF7GFP cells (Figure 3D), whereas the mesenchymal markers Vimentin and $\mathrm{N}$-cadherin were increased by S100A16 (Figure 3E). All of these data suggested that S100A16 was able to repress E-cadherin to promote EMT. 


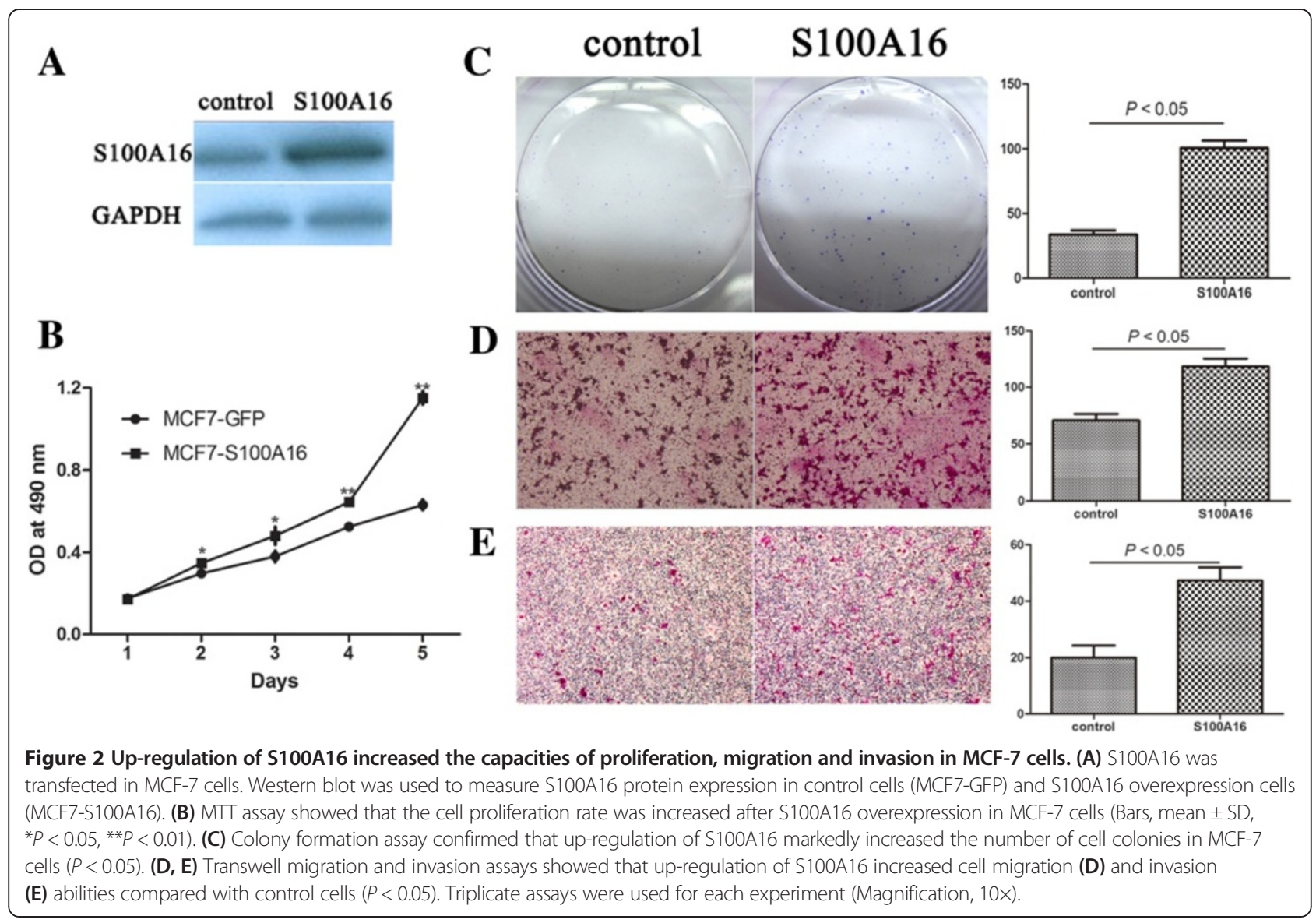

\section{Up-regulation of S100A16 increased Notch1, ZEB1 and ZEB 2 expression}

It has been reported that Notch signaling pathway is involved in EMT induction [12,13] and Notch1 is a prognosis marker for breast cancer [11]. The transcription factors ZEB1 and ZEB2 are known to repress E-cadherin in various cells [16-18]. To determine whether these transcriptional factors were regulated by S100A16, we observed that Notch1 mRNA and protein levels both were significantly enhanced in MCF7-S100A16 cells (Figure 4A and B) (similar changes were founded for T47D-S100A16 cells, data not shown). Consistently, ZEB1 and ZEB2 in MCF7-S100A16 cells were significantly higher than that in MCF7-GFP cells (Figure 4A and B). Two transcription factors ZEB1 and ZEB2 localized in the nucleus and the fluorescence signals were enhanced after S100A16 up-regulation (Figure $4 \mathrm{C}$ and D). These results demonstrated that up-regulation of S100A16 could increase transcription factors to induce EMT.

\section{Knockdown of Notch1 reversed EMT by decreasing ZEB1 and ZEB 2 expression}

As shown in Figure 4A and B, S100A16 had ability to promote EMT through regulation of ZEB1 and ZEB2. Our previous study [19] suggests Notch1 is involved in the induction of EMT in a novel breast cancer mouse model containing human breast and human bone. Here, we hypothesized that S100A16 may promote EMT through Notch1/ZEB signaling pathways. To investigate the role of Notch1, specific siRNA was used to knock down Notch1 in MCF7-S100A16 cells (Figure 5A and B). Transcription factors ZEB1 and ZEB2 were significantly reduced in mRNA and protein levels after knockdown of Notch1 (Figure 5A and B). Consequently, epithelial markers E-cadherin and $\beta$ catenin were significantly increased but mesenchymal markers Vimentin and $\mathrm{N}$-cadherin were reduced. There was no variation in the expression of S100A16 influenced by Notch1 knockdown, which indicated that S100A16 was the upstream of Notch1 (Figure 5A and B). Cell proliferation assays showed that knockdown of Notch1 decreased the proliferation of MCF7-S100A16 cells (Figure 5C). Importantly, knockdown of Notch1 decreased migration and invasion in MCF7-S100A16 cells (Figure 5D and E). These data indicated that Notch1 was a major regulator of EMT and it might be an important target site to reduce migration and invasion of breast cancer cells.

\section{S100A16 activated Notch1 transcriptional activity}

Since S100A16 was able to regulate expression of Notch1 (Figure 4A and B), we addressed the question of 


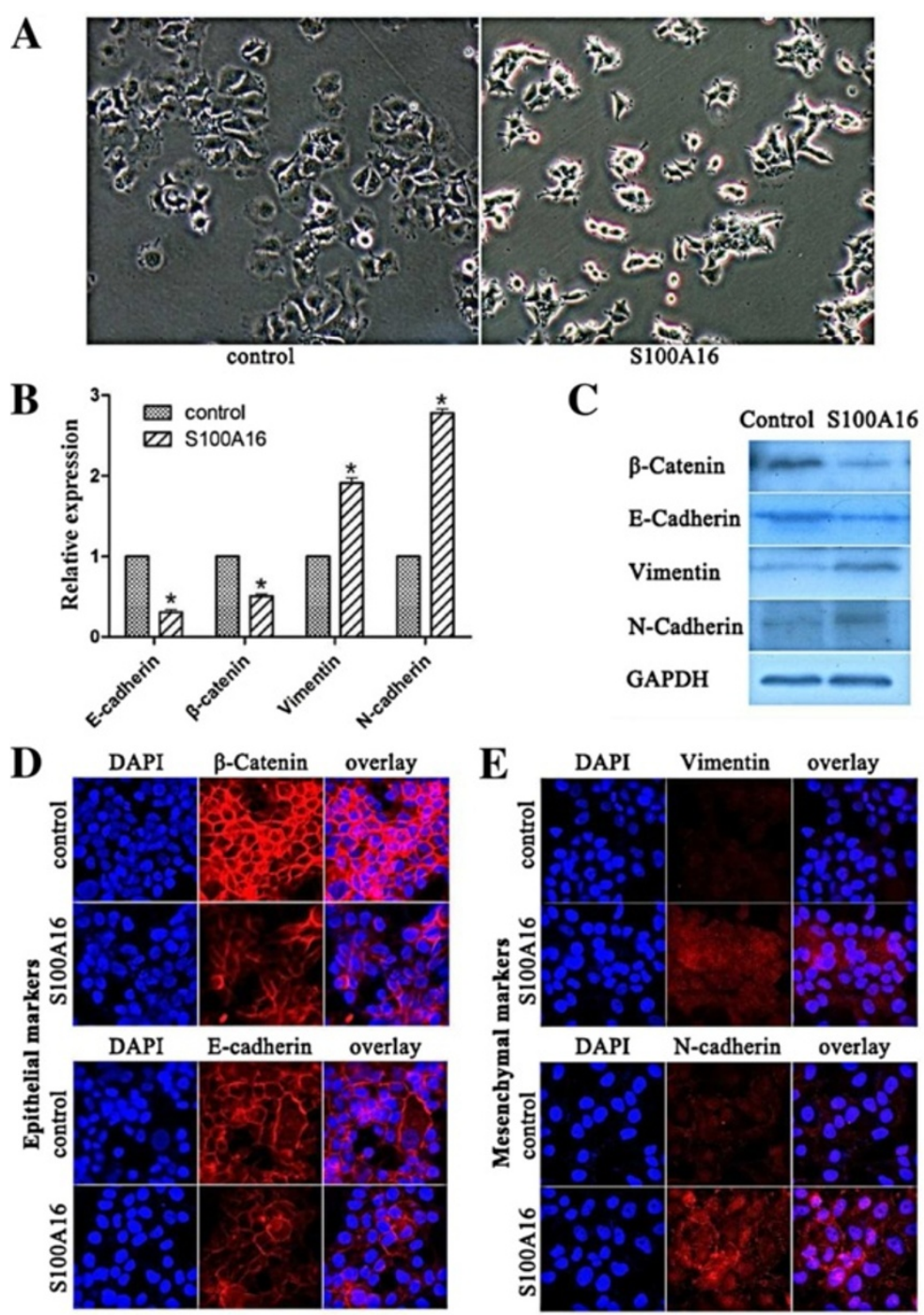

Figure 3 Overexpression of S100A16 promoted EMT in MCF-7 cells. (A) Compared with control cells (MCF7-GFP), MCF7-S100A16 cells showed spindle-like, fibroblastic morphology (10X). (B, C) qRT-PCR (B) and western blot (C) analyses showed that epithelial markers E-cadherin and $\beta$-Catenin were significantly reduced in mRNA and protein levels in MCF7-S100A16 cells compared with MCF7-GFP cells, and mesenchymal markers Vimentin and $\mathrm{N}$-cadherin were significantly up-regulated in MCF7-S100A16 cells (Bars, mean \pm SD, $P<0.05$ ). (D, E) Immunofluorescence staining was used to examine the location of epithelial and mesenchymal markers. After fixation, the celluar location of E-cadherin (red), $\beta$-catenin (red), Vimentin (red) and N-cadherin (red) were analyzed by confocal microscopy. Cell nuclei were stained with DAPI (4', 6-diamidino-2-phenylindole, blue). Immunofluorescence staining showed that both E-cadherin (red) and B-catenin (red) resided in the cell membrane and intensity of fluorescence were reduced in MCF7-S100A16 cells compared with MCF7-GFP cells (D), whereas the mesenchymal markers Vimentin (red) and N-cadherin (red) were increased by S100A16 (E).

whether it could affect the transcriptional activity of the Notch1 promoter. In S100A16-transduced MCF-7 cells, transcriptional activities were increased in four transfected plasmids (Figure 6). However, the region from -1496 to +76 had the strongest promoter activity (Figure 6). And it seemed that the region from -1496 to -998 was the core part accounting for increasing promoter activity. This result confirmed that S100A16 could regulate the transcriptional activity of the Notch1 promoter in breast cancer cells.

\section{Discussion}

S100A16 is a novel calcium-binding signaling protein of the EF-hand superfamily [7]. It has been reported that S100A16 is up-regulated in various malignant tumors [5,9], however, the role of S100A16 gene in human breast cancer is yet to be elucidated. In the present study, we first documented here that overexpression of S100A16 occurred in the human breast cancer tumors compared with the paired adjacent normal tissue. This result encouraged us to further investigate the function of S100A16 in the 


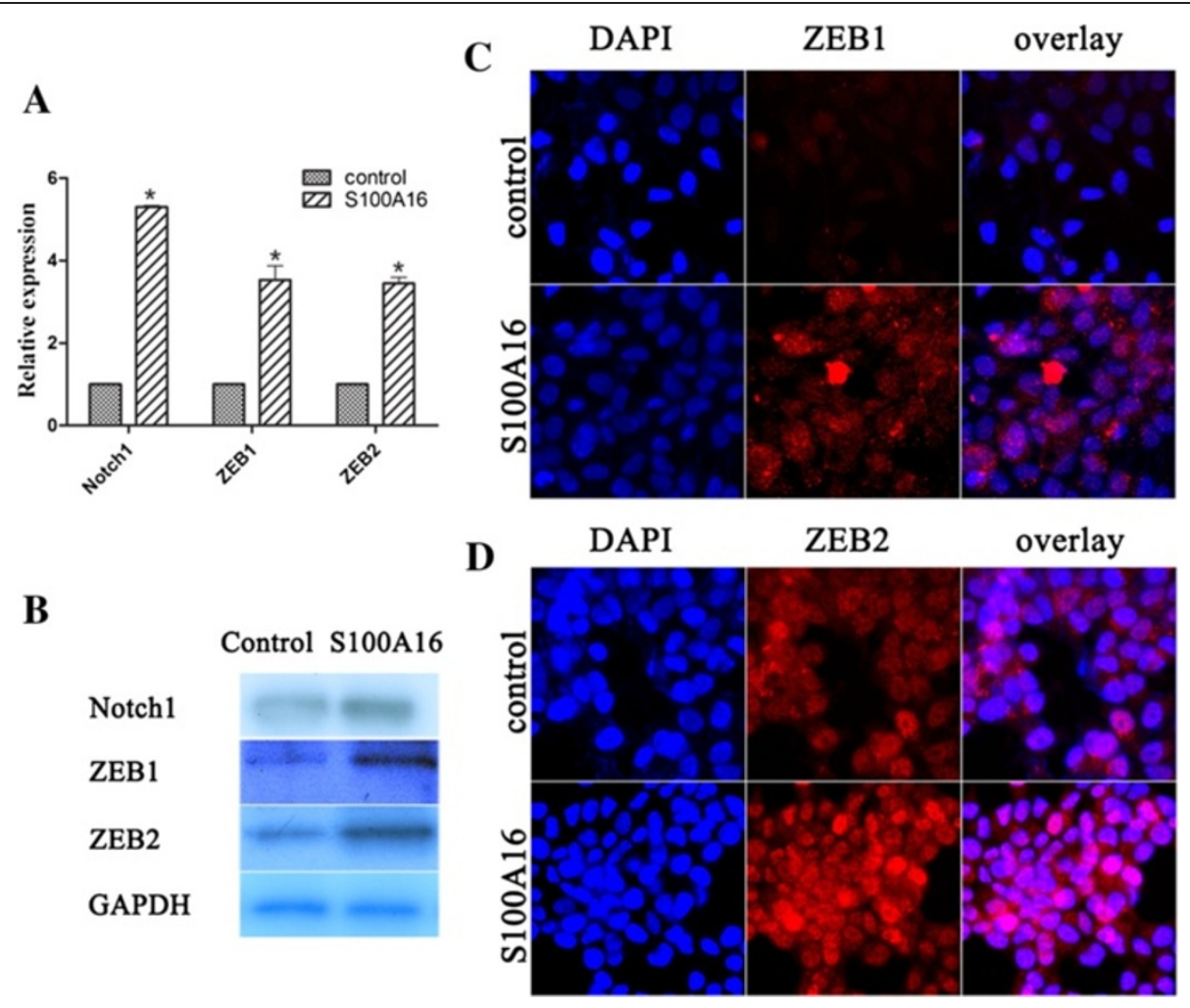

Figure 4 Up-regulation of S100A16 increased Notch1, ZEB1 and ZEB 2 expression. (A, B) qRT-PCR (A) and western blot (B) analyses showed that mRNA and protein levels of Notch 1, ZEB1 and ZEB2 were significantly enhanced in MCF7-S100A16 cells compared with control cells (Bars, mean $\pm S D, P<0.05)$. (C, D) Immunofluorescence staining was used to examine the location of two transcription factors ZEB1 and ZEB2. After fixation, the celluar location of ZEB1 (red) and ZEB2 (red) were analyzed by confocal microscopy. Cell nuclei were stained with DAPI (4', 6-diamidino-2-phenylindole, blue). Immunofluorescence staining showed that two transcription factors ZEB1 (C) and ZEB2 (D) localized in the nucleus and the fluorescence signals were enhanced after S100A16 up-regulation.

progression of breast cancer. Our previous studies suggest that S100A16 promotes adipogenesis and is involved in weight gain attenuation $[7,8]$. However, we found that overexpression of the S100A16 gene increased proliferation, migration and invasion in MCF-7 cells, and induced EMT in MCF-7 and T47D cells.

EMT is an invasive phenotype of cancer cells and involved in the events of tumor progression and metastases [10,19]. Multiple transcriptional factors are regulated in the process of EMT, demonstrating differentially in different types of cells. In our cell model, overexpression of S100A16 could up-regulate transcriptional factors ZEB1 and ZEB2, which directly repressed the expression of E-cadherin and enhanced expression of mesenchymal markers E-cadherin and vimentin. All these data indicated that the S100A16 gene might be closely associated with EMT and promote invasion and metastases during the progression of human breast cancer.

We sought to find the mechanisms how the S100A16 regulated $\mathrm{ZEB} 1$ and $\mathrm{ZEB} 2$ to promote EMT in this cell model. Many studies confirm that ZEB1 and ZEB2 are able to repress E-cadherin in various cells [16-18], and reversal of EMT requires inhibition of ZEB expression [18]. Our recent observation suggests that Notch1 is involved in the induction of EMT in a novel breast cancer mouse model [19]. In agreement with this result, overexpression of S100A16 enhanced the levels of Notch1 and regulated transcriptional activity of Notch1 in breast cancer cells. Knockdown of Notch1 decreased the levels of ZEB1 and ZEB2. Importantly, EMT was reversed after decreasing Notch1 expression in the S100A16 overexpressed cell line. All of these results first indicated that Notch1 was the upstream of ZEB and may play an important role in triggering EMT in breast cancer cells. We first demonstrated here that S100A16 overexpression induced EMT via Notch1/ZEB pathway.

It is well known that EMT is a complicated process with many pathways involving in, such as Wnt pathway, TGF- $\beta$ pathway, and Shh pathway etc. [10,20-22]. These factors interact with each other to affect the phenotype of cells. Among the tested cell lines, it was interesting to 


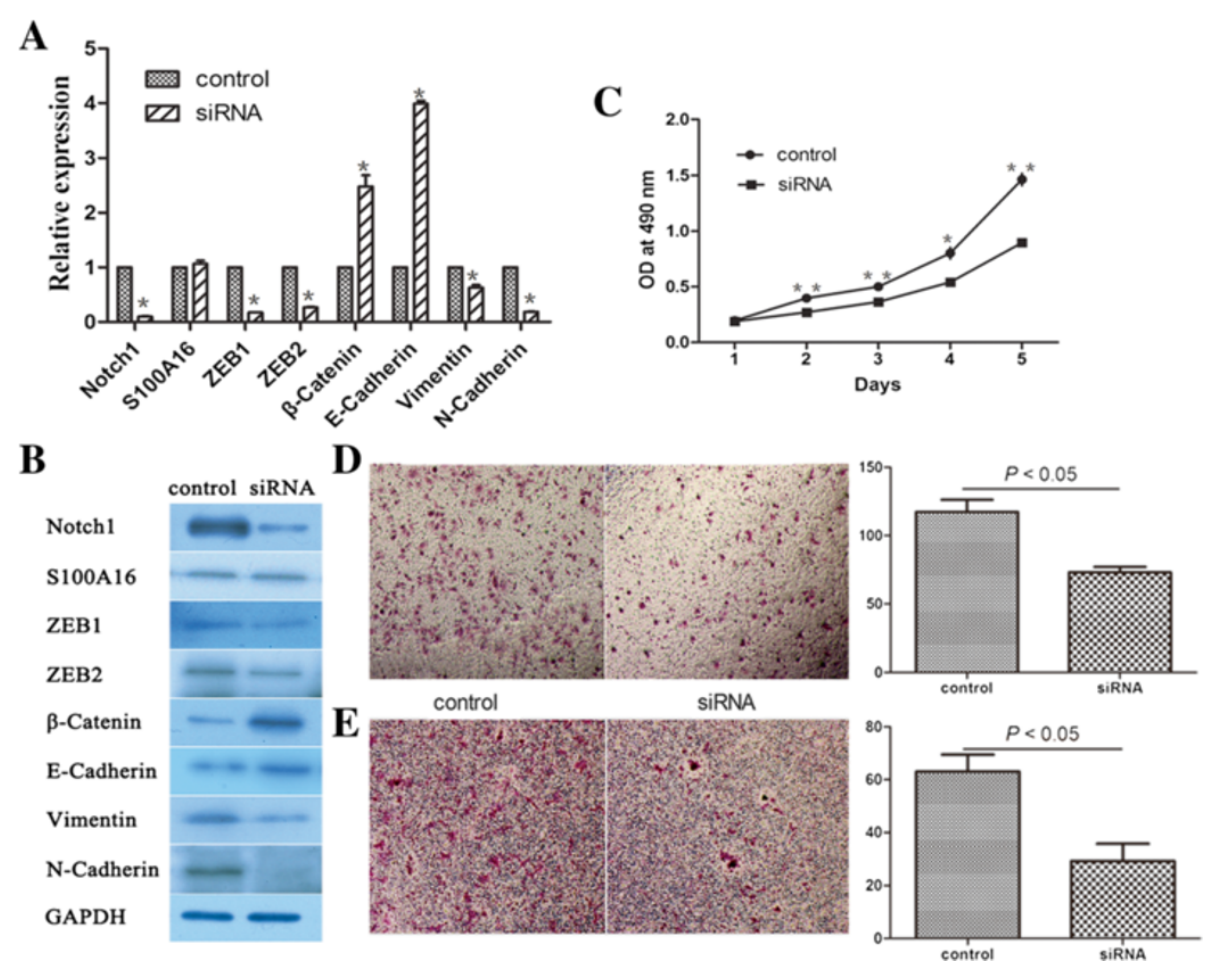

Figure 5 Knockdown of Notch1 reversed EMT by decreasing ZEB1 and ZEB 2 expression. (A, B) Specific siRNA was used to knockdown Notch1 in MCF7-S100A16 cells. GRT-PCR (A) and western blot (B) analyses showed that Notch1 was successfully knockdown. ZEB1 and ZEB2 were significantly reduced in mRNA and protein levels after knockdown of Notch1. Consequently, epithelial markers E-cadherin and $\beta$-Catenin were significantly increased but mesenchymal markers Vimentin and N-cadherin were reduced. There was no variation in the expression of S100A16 influenced by Notch1 knockdown. (Bars, mean $\pm S D, P<0.05$ ). (C) MTT assay showed that knockdown of Notch1 decreased the proliferation of MCF7-S100A16 cells (Bars, mean $\pm S D,{ }^{*} P<0.05,{ }^{*} P<0.01$ ). (D, E) Transwell migration and invasion assays showed knockdown of Notch1 decreased migration and invasion in MCF7-S100A16 cells $(P<0.05)$. Triplicate assays were used for each experiment (Magnification, 10X).

find that S100A16 protein was mainly expressed in epithelial breast cancer cell lines but not in mesenchymal breast cancer cell lines. Moreover, expression of S100A16 was detected in ER-positive cell line, HER2-positive cell line, as well as triple-negative cell line. It is unclear the association among S100A16, ER, and HER2 in breast cancer. More investigation is required to find the relationship of S100A16 with two important clinical biomarkers ER and HER2 in breast cancer. It is also under study to follow up the patients with S100A16 overexpresstion to confirm the function of S100A16 in the progression of breast cancer.

\section{Conclusions}

In summary, the current study demonstrated that overexpression of S100A16 gene promoted EMT via Notch1/ ZEB1, ZEB2/EMT pathway in breast cancer cells. These findings suggested that S100A16 could be a target for the intervention of human breast cancer.

\section{Firefly/renilla fluorescence ratio}

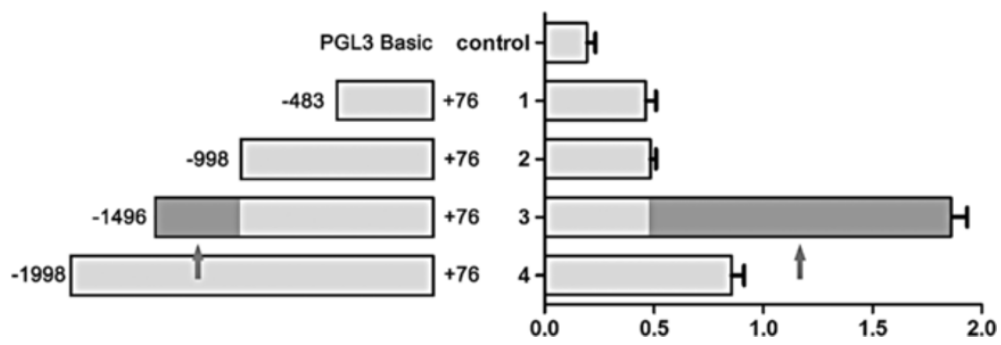

Figure 6 S100A16 activated Notch1 transcriptional activity. Dual luciferase reporter assay showed that transcriptional activities were increased in four transfected plasmids in S100A16-transduced MCF-7 cells (Bars, mean \pm SD, $P<0.05$ ). However, the region from -1496 to +76 had the strongest promoter activity. It seemed that the region from -1496 to -998 was the core part accounting for increasing promoter activity. 


\section{Additional file}

Additional file 1: Figure S1. Western blot analyses showed S100A16 protein expression was not detected in normal breast epithelial cells 184A1 and 184B5. Figure S2. Western blot analyses showed that epithelial markers E-cadherin and $\beta$-Catenin were significantly reduced in protein levels in T47D-S100A16 cells compared with T47D-GFP cells, and mesenchymal markers Vimentin and $\mathrm{N}$-cadherin were significantly up-regulated in T47D-S100A16 cells. Figure S3. Transwell migration assay showed that up-regulation of S100A16 increased cell migration abilities in T47D breast cancer cells $(P<0.05)$. Figure S4. Transwell invasion assay showed that up-regulation of S100A16 increased cell invasion abilities in T47D breast cancer cells $(P<0.05)$.

\section{Abbreviations}

ATCC: American tissue culture collection; EMT: Epithelial-mesenchymal transition; ER: Estrogen receptor.

\section{Competing interests}

The authors declare that they have no competing interests.

\section{Authors' contributions}

SW and $Y L$ have contributed to the conception and design of the study, the analysis and interpretation of data, the revision of the article as well as final approval of the version to be submitted. Wenbin Zhou, HP and XL participated in the design of the study, performed the statistical analysis, drafted and revised the article. Wenbin Zhou, HP, XL, QD, JX, LC, PF, YZ, Weidong Zhu, YX performed the experimental study. All authors read and approved the final version of the manuscript.

\section{Acknowledgement}

This work was supported in part by the National Natural Science Foundation of China $(81071753,81172505,81202077$ and 81272916), the Natural Science Foundation of Jiangsu Province (BK20141023), the Jiangsu Province's Key Provincial Talents Program (BE 2011802), the Program for Development of Innovative Research Team in the First Affiliated Hospital of NJMU (IRT-008) and a project Funded by the Priority Academic Program Development of Jiangsu higher Education Institutions (PAPD).

\section{Author details}

${ }^{1}$ Department of Breast Surgery, The First Affiliated Hospital with Nanjing Medical University, 300 Guangzhou Road, 210029 Nanjing, China. ${ }^{2}$ Department of Oncology, Lombardi Comprehensive Cancer Center, Georgetown University, Washington, DC 20057, USA. ${ }^{3}$ Department of Pathology, Nanjing Drum Tower Hospital, Nanjing University Medical School, 321 Zhongshan Road, 210008 Nanjing, China. ${ }^{4}$ Department of Urology, Zhongda Hospital Affiliated to Southeast University, Nanjing 210008, China. 5 Department of Geratology, The First Affiliated Hospital with Nanjing Medical University, 300 Guangzhou Road, 210029 Nanjing, China.

Received: 10 May 2014 Accepted: 30 September 2014

Published online: 07 October 2014

\section{References}

1. Early Breast Cancer Trialists' Collaborative Group (EBCTCG): Effects of chemotherapy and hormonal therapy for early breast cancer on recurrence and 15-year survival: an overview of the randomised trials. Lancet 2005, 365(9472):1687-1717.

2. van 't Veer LJ, Dai H, van de Vijver MJ, He YD, Hart AA, Mao M, Peterse HL, van der Kooy K, Marton MJ, Witteveen AT, Schreiber GJ, Kerkhoven RM, Roberts C, Linsley PS, Bernards R, Friend SH: Gene expression profiling predicts clinical outcome of breast cancer. Nature 2002, 415(6871):530-536

3. Chang HY, Nuyten DS, Sneddon JB, Hastie T, Tibshirani R, Sorlie T, Dai H, He YD, van't Veer $L$, Bartelink $H$, van de Rijn M, Brown PO, van de Vijver MJ: Robustness, scalability, and integration of a wound-response gene expression signature in predicting breast cancer survival. Proc Natl Acad Sci U S A 2005, 102(10):3738-3743.
4. Sturchler E, Cox JA, Durussel I, Weibel M, Heizmann CW: S100A16, a novel calcium-binding protein of the EF-hand superfamily. J Biol Chem 2006, 281(50):38905-38917.

5. Marenholz I, Heizmann CW: S100A16, a ubiquitously expressed EF-hand protein which is up-regulated in tumors. Biochem Biophys Res Commun 2004, 313(2):237-244.

6. Babini E, Bertini I, Borsi V, Calderone V, Hu X, Luchinat C, Parigi G: Structural characterization of human S100A16, a low-affinity calcium binder. J Biol Inorg Chem 2011, 16(2):243-256.

7. Liu Y, Zhang R, Xin J, Sun Y, Li J, Wei D, Zhao AZ: Identification of S100A16 as a novel adipogenesis promoting factor in 3 T3-L1 cells. Endocrinology 2011, 152(3):903-911.

8. Zhang R, Zhu W, Du X, Xin J, Xue Y, Zhang Y, Li D, Liu Y: S100A16 mediation of weight gain attenuation induced by dietary calcium. Metabolism 2012, 61(2):157-163.

9. Yao R, Lopez-Beltran A, Maclennan GT, Montironi R, Eble JN, Cheng L: Expression of S100 protein family members in the pathogenesis of bladder tumors. Anticancer Res 2007, 27(5A):3051-3058.

10. Thiery JP, Acloque H, Huang RY, Nieto MA: Epithelial-mesenchymal transitions in development and disease. Cell 2009, 139(5):871-890.

11. Yao K, Rizzo P, Rajan P, Albain K, Rychlik K, Shah S, Miele L: Notch-1 and notch-4 receptors as prognostic markers in breast cancer. Int J Surg Pathol 2011, 19(5):607-613.

12. Wu Y, Zhou BP: New insights of epithelial-mesenchymal transition in cancer metastasis. Acta Biochim Biophys Sin (Shanghai) 2008, 40(7):643-650.

13. Wang Z, Li Y, Kong D, Banerjee S, Ahmad A, Azmi AS, Ali S, Abbruzzese JL, Gallick GE, Sarkar FH: Acquisition of epithelial-mesenchymal transition phenotype of gemcitabine-resistant pancreatic cancer cells is linked with activation of the notch signaling pathway. Cancer Res 2009, 69(6):2400-2407.

14. Pan H, Zhou W, He W, Liu X, Ding Q, Ling L, Zha X, Wang S: Genistein inhibits MDA-MB-231 triple-negative breast cancer cell growth by inhibiting NF-kappaB activity via the Notch-1 pathway. Int J Mol Med 2012, 30(2):337-343.

15. Zheng MJ, Wang J, Chen YW, Xu L, Xue DD, Fu W, Zhang YF, Du Q, Zhao Y, Ling $L$, Ding Q, Liu XA, Zha XM, Zheng W, Xia TS, Wang S: A novel mouse model of gastric cancer with human gastric microenvironment. Cancer Lett 2012, 325(1):108-115.

16. Kong C, Wang C, Wang L, Ma M, Niu C, Sun X, Du J, Dong Z, Zhu S, Lu J, Huang B: NEDD9 is a positive regulator of epithelial-mesenchymal transition and promotes invasion in aggressive breast cancer. PLOS One 2011, 6(7):e22666.

17. Chua HL, Bhat-Nakshatri P, Clare SE, Morimiya A, Badve S, Nakshatri $H$ : NF-kappaB represses E-cadherin expression and enhances epithelial to mesenchymal transition of mammary epithelial cells: potential involvement of ZEB-1 and ZEB-2. Oncogene 2007, 26(5):711-724.

18. Das S, Becker BN, Hoffmann FM, Mertz JE: Complete reversal of epithelial to mesenchymal transition requires inhibition of both ZEB expression and the Rho pathway. BMC Cell Biol 2009, 10:94.

19. Xia TS, Wang GZ, Ding Q, Liu XA, Zhou WB, Zhang YF, Zha XM, Du Q, Ni XJ, Wang J, Miao SY, Wang S: Bone metastasis in a novel breast cancer mouse model containing human breast and human bone. Breast Cancer Res Treat 2012, 132(2):471-486.

20. Vincent T, Neve EP, Johnson JR, Kukalev A, Rojo F, Albanell J, Pietras K, Virtanen I, Philipson L, Leopold PL, Crystal RG, de Herreros AG, Moustakas A, Pettersson RF, Fuxe J: A SNAIL1-SMAD3/4 transcriptional repressor complex promotes TGF-beta mediated epithelial-mesenchymal transition. Nat Cell Biol 2009, 11(8):943-950.

21. Stemmer V, de Craene B, Berx G, Behrens J: Snail promotes Wnt target gene expression and interacts with beta-catenin. Oncogene 2008, 27(37):5075-5080.

22. Wang X, Zheng M, Liu G, Xia W, McKeown-Longo PJ, Hung MC, Zhao J: Kruppel-like factor 8 induces epithelial to mesenchymal transition and epithelial cell invasion. Cancer Res 2007, 67(15):7184-7193.

\section{doi:10.1186/s12929-014-0097-8}

Cite this article as: Zhou et al.: Up-regulation of S100A16 expression promotes epithelial-mesenchymal transition via Notch1 pathway in breast cancer. Journal of Biomedical Science 2014 21:97. 